\title{
Performance on steering power ratio of tracked vehicles influenced by soil parameters
}

\author{
Yuan $\mathrm{Chi}^{\mathrm{a}}$, Rongrong Zhang, Hongtao Wang and Dandan Shi \\ Northeast Agricultural University Engineering School in Harbin, China
}

\begin{abstract}
In order to study tracked vehicle adopting different steering mechanism, we use steering power ratio to evaluate its performance. The influencing factors of steering power ratio have been theoretically studied. And we designed the experimental prototype to test. We find that steering power ratio is related to vehicle parameters, resistance coefficient, turning radius, skid ratio, slip ratio and steering coefficient not only, but also it is related to soil parameters (including soil cohesion, internal friction angle of soil and shear modulus of soil). On the condition that vehicle parameters and resistance coefficient are determined, the soil shear tests were carried out, which acquired that the average moisture content of soil is $16.85 \%$, soil cohesion is $14.071 \mathrm{Kpa}$, internal friction angle of soil is $17.93^{\circ}$, and shear modulus of soil is $0.00027 \mathrm{~m}$. Through being researched, steering power ratio is related to turning radius, skid ratio and slip ratio, which has the important significance on the study of steering power ratio.
\end{abstract}

\section{Introduction}

Tracked vehicle can be steered in small plot by differential steering, further more, the turning radius can be zero. There is no brake power loss and being steered in any circle when differential steering, compared with the clutch steering. When tracked vehicle steering or straight driving on the soil, it would causes skid and slip phenomenon. So we have to analyze the mechanical properties of the soil. When tracked vehicle walking on soft soil [1-2], the tangential driving force of tracked vehicle is mainly due to the occurrence of shear of the soil, that is the shear of the soil pushing the vehicle ahead. Based on the point of soil mechanics, the pushing is a character of soil, and it has the function of making the vehicle going ahead. Soil would slide for resisting the shear deformation. The shear characters of soil directly influence the driving force of the vehicle and the occurrence of skid and slip. Lianhua Gao [3-4] has studied steering power ratio of military tracked vehicles, and he found that steering power ratio is affected by soil conditions; Tran Dang Thai [5] has analyzed steering characteristics of tracked vehicle. Through the establishment of mechanical model and software analysis, he obtained the relationship curve between slip ratio, driving force and turning radius of tracked vehicle; The theory of classical mechanics [6-7] has described the relationship between tangential driving force and shear stress of soil, tangential driving force and skid ratio, shear stress of soil and displacement, vertical pressure and sinkage. It said that in the case of same skid ratio, the ground that has good adhesion performance can provide large tangential driving force. But, the study how the skid and slip impact on steering power ratio is very few. According to the theory of soil mechanics, the paper makes a theoretical analysis on the influencing factors of steering power ratio, and on the basis of differential steering characteristics of tracked vehicle, we deduce the expression of steering power ratio. At the same time on the condition that vehicle parameters and resistance coefficient are determined, through the soil shear tests we got the values of soil parameter and then got the formula of steering power ratio, turning radius, skid ratio and slip ratio. They can provide a theoretical basis for evaluating the different steering mechanism of tracked vehicle.

\section{The experimental prototype and the vehicle's steering performance}

\subsection{The structure principle of experimental prototype}

Design schematic of the differential steering mechanism is shown in figure 1.

This mechanism is composed by three planetary trains. Including two power input sides $\mathrm{A}$ with $\mathrm{C}$, and two power output half shafts $\mathrm{O} 1$ with $\mathrm{O} 2$. Power source of $\mathrm{A}$ comes from transmission, and $\mathrm{C}$ comes from hydraulic motor.

\footnotetext{
${ }^{\mathrm{a}}$ Yuan Chi: cy207@126.com.org
} 


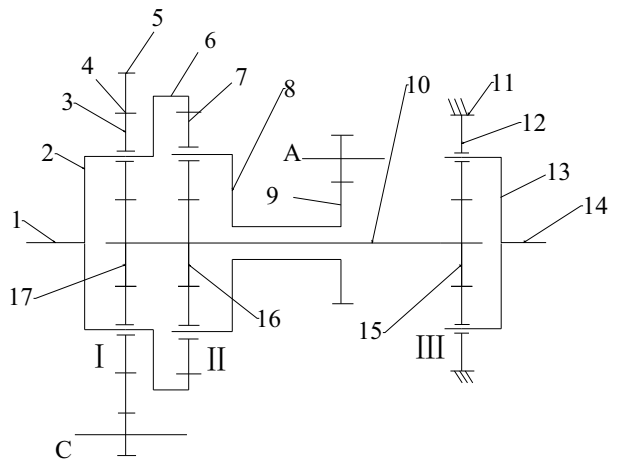

Note: A-Output shaft of transmission; C- Output shaft of hydraulic steering motor; I , II ,III planetary gear trains.

1. Left half shaft 2,8,13. Carrier 3,7,12. Gear (planet) 4,6,11. Gear (ring) 5. Gear (steering power input) 9. Gear (straight power input) 10. Intermediate shaft 14. Right half shaft $15,16,17$. Gear (sun)

Figure 1. Principle of the different steering mechanism

\subsection{The design of the experimental prototype}

In figure 1 , the power input of $\mathrm{A}$ and $\mathrm{C}$ come from two three-phase asynchronous motors driven by chain transmission respectively. Both electric motors are controlled by variable-frequency converters, and are connected to the reversing switches, respectively. Forward, backward, turning left, turning right, speed up and down of experimental prototype can be controlled by variable-frequency converters and reversing switches. When straight driving, only $\mathrm{A}$ is driven, the speed is controlled by frequency converter, and forward or backward are controlled by reversing switch; While steering, both $\mathrm{A}$ and $\mathrm{C}$ are driven at the same time, and the size of turning radius is controlled by two frequency converters together, but the direction of rotation is controlled by the reversing switch that connected $\mathrm{C}$. When turning at neutral steering, only the hydraulic motor works.

\subsection{The vehicle's steering performance and evaluation items}

Steering performance is an important item to evaluate the performance of tracked vehicle, especially the performance of the steering mechanism of the tracked vehicle. The performance of steering mechanism is evaluated mainly by the minimum turning radius of tracked vehicle, turning angular velocity, the growing degree of engine load, straight motion stability, etc. In this paper, we mainly researched the growing degree of engine load.

\section{Theoretical researches}

\subsection{Steering power ratio}

In the same soil condition, the loads are identical (that is driving force of the vehicle in steady steering is equal to that in uniform linear motion). The ratio of engine power (that is the power ratio of tracked vehicle in steady steering and uniform linear motion) is called steering power ratio.

For the differential steering mechanism, the speed increment of outside track is equal to the reduction of interior track. The velocity of mass center is constant when tracked vehicle steering. So steering power ratio of differential steering mechanism can be expressed as follow [8-9]:

$$
\xi=\frac{P_{2}}{P_{1}}=\frac{f G v+M_{\mu} \omega}{f G v}=1+\frac{M_{\mu} \omega}{f G v}
$$

Where:

$P_{2}=$ power consumed when steering, W;

$P_{1}=$ power consumed when straight driving, $\mathrm{W}$;

$f=$ resistance coefficient;

$G=$ weight of the vehicle, $\mathrm{N}$;

$v=$ velocity of mass center, $\mathrm{m} / \mathrm{s}$;

$M \mu=$ steering resistance torque, $\mathrm{N} \cdot \mathrm{m}$;

$\omega=$ rotational angular velocity, $\mathrm{rad} / \mathrm{s}$.

When tracked vehicles in stable steering, the steering resistance torque is equal to the steering torque $M_{\mu}=M_{z}$. The reason of tracked vehicle steering is that the driving forces of the outside track and interior are different. So steering torque can be expressed as follow:

$$
M_{z}=\frac{B}{2}\left(F_{t o}-F_{t i}\right)
$$

Where:

$B=$ track gauge;

$F_{t o}=$ driving force of outside track, N;

$F_{t i}=$ driving force of interior track, N.

The rotational angular velocity can be expressed as follow:

$$
\omega=\frac{v}{R}
$$

Where $R$ is turning radius, $\mathrm{m}$.

The driving forces on both sides of the track [10] can be expressed as follow:

$$
\begin{aligned}
& F_{t o}=\left(b L C+\frac{G}{2} \tan \varphi\right)\left[1-\frac{K}{i_{o} L}\left(1-e^{-\frac{i_{o} L}{K}}\right)\right] \\
& F_{t i}=\left(b L C+\frac{G}{2} \tan \varphi\right)\left[1-\frac{K}{i_{i} L}\left(1-e^{-\frac{i_{i} L}{K}}\right)\right]
\end{aligned}
$$

Where:

$b=$ track width, m;

$L=$ track length, $\mathrm{m}$

$C=$ soil cohesion, $\mathrm{kPa}$;

$\varphi=$ internal friction angle of soil, $\left(^{\circ}\right)$; 
$K=$ shear modulus of soil, $\mathrm{m}$.

By the formulas (1), (2), (3), (4) and (5), we can get the formula of steering power ratio as follow: $\xi=1+\frac{B / 2}{R} \cdot \frac{\left(b L C+\frac{G}{2} \tan \varphi\right)\left[\frac{K}{i_{i} L}\left(1-e^{-\frac{i_{i} L}{K}}\right)-\frac{K}{i_{o} L}\left(1-e^{-\frac{i_{o} L}{K}}\right)\right]}{f G}$

Through the formula (6), we can know that steering power ratio is related to soil parameters (soil cohesion $C$, internal friction angle of soil $\varphi$ and shear modulus $K$ ), vehicle parameters ( track width $b$, ground contact length of track $L$, track gauge $B$, weight of the vehicle $G$ ), resistance coefficient $f$, turning radius $R$, skid ratio $i_{o}$ and slip ratio $i_{i}$.

\subsection{Shear strength of soil}

The shear strength of soil [11] is an ability of resisting shear and destruction, and it is one of the most important mechanical characters of soil. All of these questions including the foundation bearing capacity in civil engineering, the pressure of soil dam and soil slope stability are related to the shear strength of soil. According to Coulomb's law, the shear strength of soil is proportional to vertical stress on the shear plane. Its essence is sliding friction between soil particles and friction resistance produced by the concave and convex. Its value depends on the surface roughness of the soil particle, density and size of soil particles. The shear strength of clayey soil is composed of two parts. One part is the friction force, and the other part is the adhesive force between soil particles. The shear strength of soil can be expressed as follow:

$$
\tau_{f}=C+\sigma \cdot \tan \varphi
$$

Where:

$\tau_{f}=$ shear strength of soil, $\mathrm{kPa}$;

$\sigma=$ vertical strength acting on the shear plane, $\mathrm{kPa}$.

Based on calculating an integral of the single track contact area and the formula (7), we can get the maximum driving force on both sides of the track as follow:

$$
F_{t o}=F_{t i}=b L C+\frac{G}{2} \tan \varphi
$$

Destruction of soil is shear destruction, and the reason is that the strength of soil is not enough to resist shear stress on the shear plane. The shear plane along the shear stress direction has a drastic deformation, and then it will be destroyed. This surface is called shear surface. The force produced by the interaction of tracked vehicle and soil in the horizontal direction is called the shear stress of soil. The maximum shear stress is equal to the shear strength. Relation between shear stress and shear displacement [12] can be expressed as follow:

$$
\tau=\tau_{f}\left(1-e^{-\frac{j}{K}}\right)
$$

After taking the derivative of formula (9), we can get another formula as follow:

$$
-\ln \left(1-\tau / \tau_{f}\right)=\frac{j}{K}=\frac{1}{K} j
$$

\section{Experimental study}

\subsection{Vehicle coefficient \\ parameters and resistance}

The prototype experiments are carried out on the soft soil. Relying on table look-up [13], we can know that resistance coefficient of soft soil is 0.1 . So the driving resistance of tracked vehicle is $163.66 \mathrm{~N}$. In the test, the experimental data are as follows: track width is $0.15 \mathrm{~m}$, track length is $0.715 \mathrm{~m}$.

\subsection{Soil shear tests}

Through the soil shear tests [14], we can measure soil cohesion $C$, internal friction angle of soil $\varphi$ and shear modulus $\mathrm{K}$. Then we can quantize the influencing factors of steering power ratio. It has a great significance for the study of steering power ratio.

In the test, shear stress can be expressed as follow:

$$
\tau=D \cdot Z
$$

Where:

$D=$ correction coefficient of proving ring, the value of it is $2.0348 \mathrm{kpa} / 0.01 \mathrm{~mm}$;

$Z$ =reading of dial indicator in the proving ring, $(\times 0.01 \mathrm{~mm})$.

In the test, the shear displacement can be expressed as follow:

$$
j=Y-Z
$$

Where: $Y=$ reading of dial indicator, $(\times 0.01 \mathrm{~mm})$.

In the test, we use the fast shear test [15] to measure the soil parameters, and the instrument type is ZJ straincontrolled direct shear apparatus.

The experimental procedure is as follows:

(1) Cutting soil samples. We put the normal cutting ring on the soil, and the blade towards to down. Then we cut the undisturbed soil and mark them as sample 1, 2, 3 and 4. Use the automatic tester of soil moisture to measure six times in each sample. The experimental data obtained are as follow: The average moisture content of sample 1 is $16.67 \%$, sample 2 is $16.32 \%$, sample 3 is 
$18.57 \%$, and sample 4 is $15.85 \%$. So the average moisture content in this test is $16.85 \%$.

(2) Installation and debugging. At first, we rotate the hand wheel of adjusting lever in a counterclockwise direction. The purpose of it is to make the central shaft rise to the top. Then we adjust the counter weight so that the level is horizontal. Install 2 dial indicators, and then check them to touch.

(3) Installing the samples. When the upper and lower shear box overlap, we insert the fixing pin. Put a porous stone in the lower box, and put a piece of filter paper on the stone. Put the blade of cutting ring upwards, and ensure that it will be aligned with the upper shear box. Put a piece of wax papers on the soil sample, and then pile it into the shear box by bulldozing device. After a series of work having been completed, we remove the cutting ring, and put another porous stone on the filter paper. Then put cover, steel ball and framework on it in turn. At last, we adjust the framework to ensure the gap between the ball and the framework is $1-3 \mathrm{~mm}$.

(4) After acting a vertical pressure, we must immediately pull out the pin fixed on the shear box. We need carry out four times shear tests, and the pressures are $50,100,200,300 \mathrm{kPa}$ respectively. In the test, we turn the hand wheel at a speed of $6 \mathrm{rad} / \mathrm{min}$ to ensure soil samples are destroyed in $3 \sim 5$ minutes. And each turning we need write down the readings of 2 dial indicator and the number of hand wheel. When the readings do not increase any longer or start decreasing, the tests can be stopped. In this condition we think the samples have been destroyed. Comparison before applying vertical pressure and after are as shown in figure 2 and figure 3.

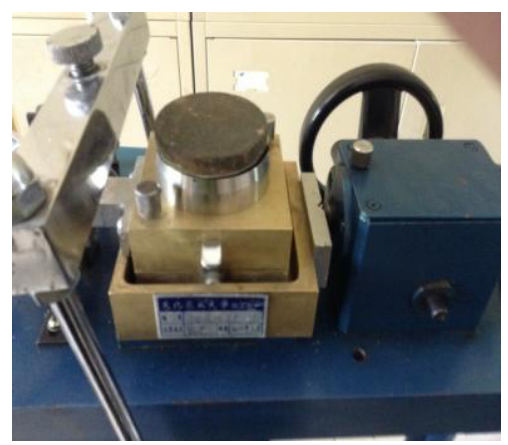

Figure 2. Before applying vertical pressure sample

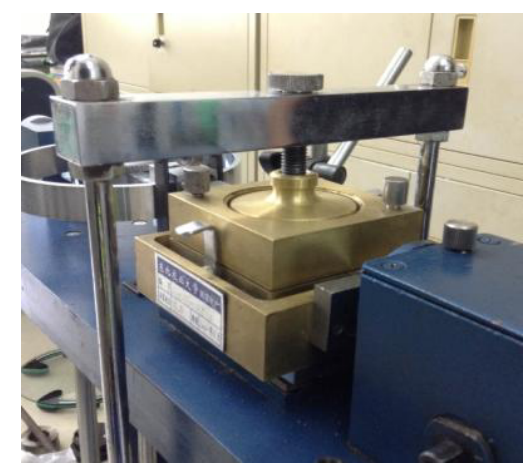

\subsection{Analysis of experimental results}

When pressures are $50,100,200,300 \mathrm{kPa}$, we get the maximum shear strength are $0.14 \mathrm{kPa}, 0.37 \mathrm{kPa}, 0.54 \mathrm{kPa}$ and $0.7 \mathrm{kPa}$ respectively. Based on the experimental data, we get the fitting curve is shown in figure 4 , and the formula of $\tau_{f}$ and $\sigma$ can be expressed as follow:

$$
\tau_{f}=14.071+0.3235 \sigma
$$

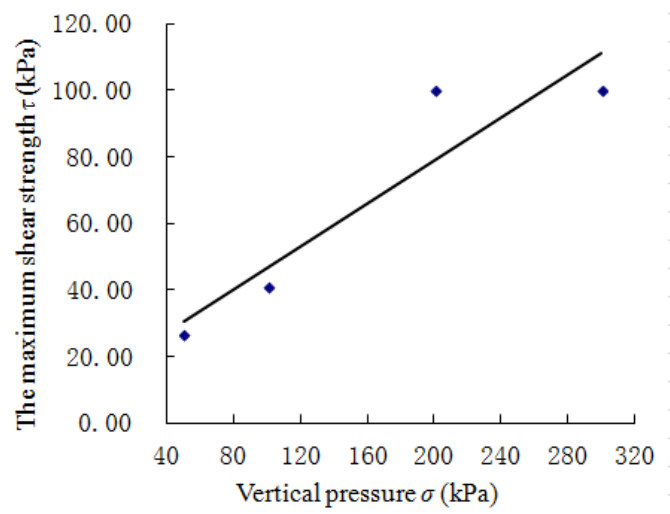

Figure 4. Relationship between shear strength and vertical pressure

According to the formula (13), we can know that soil cohesion is $14.071,(\mathrm{kPa})$, and internal friction angle is 17.92639 17.93, $\left(^{\circ}\right)$.

The fitting curves of four kinds of different vertical pressure are shown in figure 5, figure 6 , figure 7 and figure 8 .

The fitting formulas of four kinds of different vertical pressure are respectively expressed as follow:

$$
\begin{aligned}
& -\ln \left(1-\tau / \tau_{f}\right)_{1}=3.7003 j_{1} \\
& -\ln \left(1-\tau / \tau_{f}\right)_{2}=2.7621 j_{2} \\
& -\ln \left(1-\tau / \tau_{f}\right)_{3}=3.4523 j_{3} \\
& -\ln \left(1-\tau / \tau_{f}\right)_{4}=6.8565 j_{4}
\end{aligned}
$$

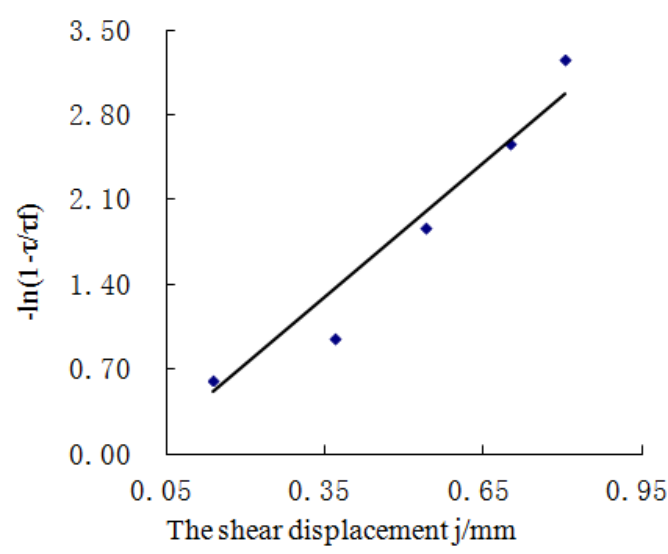

Figure 5. 50kPa $\ln \left(1-\tau / \tau_{f}\right)-j$ fitting figure

Figure 3. After applying vertical pressure sample 


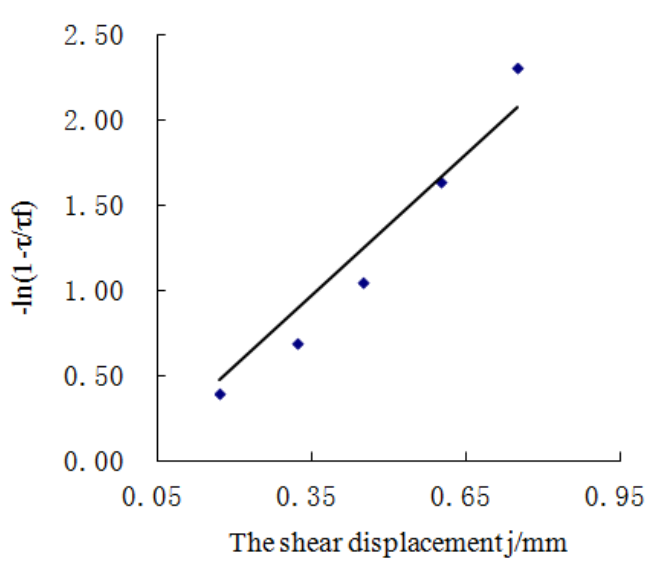

Figure 6. $100 \mathrm{kPa} \ln \left(1-\tau / \tau_{f}\right)-j$ fitting figure

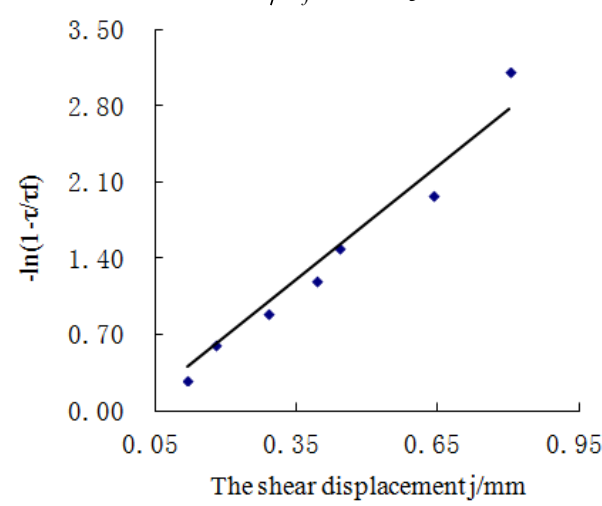

Figure 7. 200kPa $\ln \left(1-\tau / \tau_{f}\right)-j$ fitting figure

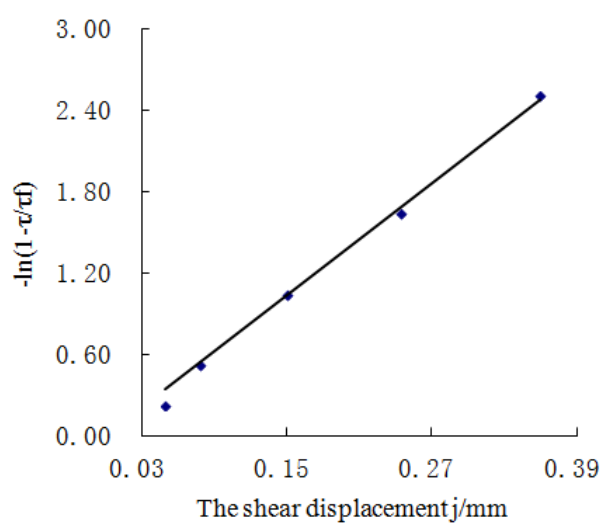

Figure 8. 300kPa $\ln \left(1-\tau / \tau_{f}\right)-j$ fitting figure

According to the formulas (14), (15), (16) and (17), we can know the following information: When vertical pressure is $50 \mathrm{kPa}, \frac{1}{K_{1}}=3.7003 \mathrm{~mm}$, shear modulus of soil is $0.00027 \mathrm{~m}$; When vertical pressure is $100 \mathrm{kPa}$, $\frac{1}{K_{2}}=2.7621 \mathrm{~mm}$, shear modulus of soil is $0.00036 \mathrm{~m}$;

When vertical pressure is $200 \mathrm{kPa}, \frac{1}{K_{3}}=3.4523 \mathrm{~mm}$, shear modulus of soil is $0.00029 \mathrm{~m}$; When vertical pressure is $300 \mathrm{kPa}, \frac{1}{K_{4}}=6.8565 \mathrm{~mm}$, shear modulus of soil is $0.00015 \mathrm{~m}$. So the average value of shear modulus is $K=0.00027 \mathrm{~m}$.

We finally get the maximum driving force on both sides of the track is as follow:

$$
F_{t o}=F_{t i}=b L C+\frac{G}{2} \tan \varphi \approx 266.3739 \mathrm{~N}
$$

When moisture content of soil is $16.85 \%$, we get the maximum pushing force provided by soil (that is the maximum driving force on both sides of the track) is $266.3739 \mathrm{~N}$.

According to the formulas (4), (5) and (18), we can get the relationship between driving force on both sides of the track, slip ratio and skid ratio can be expressed as follow:

$$
\begin{aligned}
& F_{t o}=266.3739\left[1-\frac{0.000356}{i_{o}}\left(1-e^{-\frac{i_{o}}{0.000356}}\right)\right] \\
& F_{t i}=266.3739\left[1-\frac{0.000356}{i_{i}}\left(1-e^{-\frac{i_{i}}{0.000356}}\right)\right]
\end{aligned}
$$

According to the formulas (19) and (20), we can know that the driving force on both sides of the track would change as slip ratio and skid ratio.

At last, steering power ratio can be expressed as follow:

$$
\xi=1+0.0002 \frac{\frac{1-e^{-\frac{i_{i}}{0.000356}}}{i_{i}}-\frac{1-e^{-\frac{i_{o}}{0.000356}}}{i_{o}}}{R}
$$

Based on the formula (21), we can know the value of steering power ratio is influenced by ground conditions. On different conditions, resistance coefficients are different, and the values of soil parameters obtained by the soil shear tests are different. So the formula of steering power ratio may be different. In our test, according to the soil shear tests, we quantify the influencing factors of steering power ratio. On the condition that vehicle parameters, resistance coefficient and soil parameters are determined, we get the conclusion that steering power ratio is affected by slip ratio, skid ratio and turning radius.

\section{Conclusion}

Steering power ratio is affected by soil parameters, vehicle parameters, resistance coefficient, turning radius, slip ratio and skid ratio. Soil conditions are determine slip and skid on both sides of the track, soil pushing force and steering power ratio. On the condition that vehicle parameters and resistance coefficient are determined, we quantify the influencing factors of steering power ratio by the soil shear tests, and we get the relation between steering power ratio of tracked vehicle adopting different steering mechanism, turning radius, slip ratio and skid ratio. It combined with the minimum turning radius, rotational angular velocity and straight motion stability studied by previous research group, the results can establish a foundation for the study of tracked vehicle adopting different steering mechanism, and enrich the theory of tracked vehicle. 


\section{References}

1. Lu Jinjun, Wei Laisheng, Zhao Taoshuo. Agricultural Technology \& Equipment. 8 (2007)

2. S Hutangkabodee, Y H Zweiri, L D Seneviratne. Validation of soil parameter identification for trackterrain interaction dynamics. 11, 3 (2007)

3. Fang Zhiqiang, Gao Lianhua, Wang Hongyan. Journal of Academy of Armored Force Engineering. 19, 2 (2005)

4. Song Haijun, Gao Lianhua, Li Jun. Vehicle \& Power Technology. 105, 1 (2007)

5. Tran Dang Thai, Tatsuro Muro. Journal of Terramechanics. 36, 3 (1999)

6. Chen Bingcong. China agricultural machinery press (1981)

7. M.G. Bekker. China machine press. (1978)

8. Chi Yuan, Jiang Enchen. Transactions of the Chinese Society of Agricultural Machinery. 39, 8 (2008)

9. Chi Yuan. Transactions of the CSAE. 28, 18 (2012)

10. Al-Milli, Seneviratne, Lakmal D. Journal of Terramechanics. 47 (2010)

11. Yang Shimin, Fu Xiangru. China communications press. (2010)

12. Dror Rubinstein, James L, Coppock. Journal of Terramechanics. 44 (2007)

13. Chi Yuan. Chemical industry press. (2003)

14. Wang Wei, Li Xiaoyu, Wang Zhuanwei. Agricultural research in the arid areas. 20, 1 (2002)

15. Hu Qizhi, Zhou Zheng, Xiao Benlin. Soil engineering and foundation. 24, 5 (2010) 